\title{
Immediate and Longer-Term Effects of An Intensive Adolescent Cooking Intervention on Mental Well-Being and Cooking Self-Efficacy, Attitudes and Involvement ${ }^{\dagger}$
}

\author{
Sarahmarie Kuroko ${ }^{1}$, Katherine Black ${ }^{1}$, Themis Chryssidis ${ }^{2}$, Rosie Finigan ${ }^{1}$, Callum Hann ${ }^{2}$, \\ Jill Haszard 1, Rosalie Jackson 1, Katherine Mahn 1, Caleb Robinson 1, Carla Thomson 1, \\ Olivia Toldi ${ }^{1}$ and Paula Skidmore ${ }^{1, *}$ \\ 1 Department of Human Nutrition, University of Otago, Dunedin 9016, New Zealand; \\ sarahmarie.kuroko@gmail.com (S.K.); katherine.black@otago.ac.nz (K.B.); rosiefinigan@gmail.com (R.F.); \\ jill.haszard@otago.ac.nz (J.H.); rosie_jackson@hotmail.com (R.J.); mahka292@student.otago.ac.nz (K.M.); \\ caleb.robinson@otago.ac.nz (C.R.); carla.thomson@otago.ac.nz (C.T.); oliviatoldi@gmail.com (O.T.) \\ 2 Sprout Cooking School and Health Studio, Hilton SA 5033, Australia; themis@sprout.edu.au (C.T.); \\ callum@sprout.edu.au (H.C.) \\ * Correspondence: paula.skidmore@otago.ac.nz \\ + Presented at the 2018 Nutrition Society of New Zealand Annual Conference, Auckland, New Zealand, 28- \\ 30 November 2018.
}

Published: 12 March 2019

Background: Cooking more frequently is associated with a healthier diet. However, reported decreases in adolescents' cooking opportunities may contribute to poorer health outcomes. Few cooking intervention studies have used a randomised control (RCT) design or longer-term followups. Additionally, cooking more often is associated with better mental well-being among teens but this has not been examined experimentally. Our objective was to investigate whether a cooking intervention affects cooking-related outcomes and mental well-being among adolescents.

Methods: A 5-day intensive holiday cooking program, followed by 6 weeks of weekly recipe and ingredient kits with a Facebook support group, was tested using an RCT design with a 12-month follow-up. Adolescents aged 12-15 y ( $n=118 ; 64 \%$ female) completed baseline, post-intervention and 12-month questionnaire measures of mental well-being, cooking attitudes, cooking self-efficacy and current cooking involvement. Between-group differences accounting for baseline were estimated using repeated-measures mixed regression models.

Results: Post-intervention changes were significantly higher in the intervention group for mental well-being (4.7\% higher, 95\%CI [0.1-9.3], $p=0.04)$, home cooking involvement (0.4 times/week, 95\%CI [0.1-0.7], $p=0.003$ ), cooking self-efficacy (general: $14.0 \%, 95 \%$ CI [7.3-20.6], specific: $23.1 \%, 95 \% \mathrm{CI}$ [20.6-25.7], both $p<0.001)$, and positive cooking attitude $(11.5 \%$, 95\%CI [9.7-13.4], $p<0.001)$. Differences were maintained at 12 months for self-efficacy and attitude only (general cooking selfefficacy: 7.7\%, 95\%CI [3.4-12.1]; specific cooking self-efficacy: $15.0 \%$, 95\%CI [11.8-18.2], both $p<0.001$; positive cooking attitude: $2.3 \%, 95 \% \mathrm{CI}[0.1-4.4], p=0.04)$. This study was not sufficiently powered for subgroup analyses, however exploratory analyses suggested possible effect modification by socioeconomic status, weight status or teen cooking involvement.

Conclusions: Cooking interventions may increase cooking behavior and improve mental wellbeing during support phases of interventions, however cooking attitudes and self-efficacy are more likely to be maintained longer-term. Future research should investigate what factors help maintain cooking involvement and mental well-being among adolescents. 
Funding: Lotteries Health New Zealand, Foodstuffs Community Trust.

(C) 2019 by the authors. Licensee MDPI, Basel, Switzerland. This article is an open access article distributed under the terms and conditions of the Creative Commons Attribution (CC BY) license (http://creativecommons.org/licenses/by/4.0/). 